\title{
Post Mortem Ultrasound and Computed Tomography Findings of an Extraluminal Urinary Bladder Leiomyoma in a Dog
}

\author{
Bruno Ferrante', Carina Outi Baroni', Caterina Muramoto', Igor Almeida dos Santos', \\ Hock Gan Heng' \& Ana Carolina Brandão de Campos Fonseca Pinto ${ }^{1}$
}

\begin{abstract}
Background: Neoplasia of the urinary bladder is common in dogs, accounting approximately 0.5 to 1.0 percent of all neoplasms. Most of the neoplasia of the urinary bladder is epithelial in origin and only $10 \%$ of urinary bladder neoplasms in dogs are from mesenchymal origin, of which the most frequent types are leiomyoma / leiomyosarcoma, and hemangioma / hemangiosarcoma. Virtual autopsy refers to the postmortem use of radiology, ultrasound and cross-sectional imaging prior to conventional necropsy. This paper reports the detection of a rare extra-luminal urinary bladder mass diagnosed as leiomyoma with a virtual autopsy techniques.

Case: A 16-year-old male Schnauzer had previous history of seizure and no complains related to the urinary system. The animal was treated symptomatically to the neurological signs and responded to medical treatment. Nine weeks later from the first visit to the hospital the dog was found dead at home. Then postmortem ultrasound and computed tomography of the abdomen were performed. Postmortem ultrasound revealed a homogenously hypoechoic, rounded and slightly irregularly marginated mass located externally but adjacent to the left cranial wall of the urinary bladder and appears to extend from its serosal margins. Postmortem computed tomography was performed after postmortem ultrasound. A pedunculated homogenous soft tissue attenuating mass was located at the left lateral aspect of the urinary bladder and extended cranially. It had a stalk that connected to the left lateral wall of the urinary bladder. A partial necropsy of the abdomen was done just to examine the mass. A round extraluminal, pedunculated mass was observed at the left lateral aspect of the urinary bladder wall. It was pale pink on the outside and white inside, with a soft to firm consistency. The lumen and mucosal surface of the urinary bladder was smooth and regular. The histology of the mass revealed a densely cellular neoplastic proliferation, expansive, composed of spindle-shaped cells with moderate to large eosinophilic cytoplasm, sometimes wavy and with indistinct edges. The nuclei were large, oval to flattened, with dense chromatin and inconspicuous nucleoli. Anisocytosis and anisokariosis were discrete and no mitotic figures were observed. The arrangement consisted of dense, irregular and multidirectional bundles and the stroma was scarce. The mass was histologically confirmed as leiomyoma.

Discussion: In this case, we performed postmortem ultrasound and computed tomography as part of a virtual necropsy study and in both modalities the urinary bladder mass was able to be identified, followed by a partial necropsy to further investigate the nature of the mass and to collect a sample to obtain the histological diagnosis. A few of the disadvantages of the postmortem ultrasound and computed tomography specially in this case were the lack of color Doppler investigation on ultrasound and the lack of evaluation of the patter of contrast enhancement on computed tomography. These techniques could have added important information related to the vascularity characteristics of the mass in a live patient. This is the first case report in veterinary medicine that describes an extra-luminal pedunculated urinary bladder leiomyoma in a canine patient, and it is emphasized the approach by postmortem ultrasound, postmortem computed tomography and conventional necropsy findings to reach the definitive diagnosis.
\end{abstract}

Keywords: virtual necropsy, urinary neoplasm, post mortem imaging diagnosis. 
B. Ferrante, C.O. Baroni, C. Muramoto, et al. 2017. Post Mortem Ultrasound and Computed Tomography Findings of

\section{INTRODUCTION}

Neoplasia of the urinary bladder is common in dogs, accounting approximately 0.5 to 1.0 percent of all canine neoplasms [4]. Most of the neoplasia of the urinary bladder is epithelial in origin and only $10 \%$ of urinary bladder neoplasms in $\operatorname{dog} s$ are from mesenchymal origin, of which the most frequent types are leiomyoma / leiomyosarcoma, and hemangioma / hemangiosarcoma [2,4]. Urinary bladder leiomyoma / leiomyosarcoma are rare and only represent 0.5 to 4 percent of all canine urinary bladder neoplasm [7,9].

Canine leiomyoma / leiomyosarcomas has been described as a single, rounded and welldefined intraluminal mass, with mixed echogenicity and lack of detectable blood flow on color Doppler mode [2,3]. In human medicine, leiomyomas are classified according to the anatomic location of the mass as endovesical, intramural and extravesical [6]. Intramurals leiomyosarcoma and leiomyoma have been reported in humans $[1,10]$. The reported $\mathrm{HU}$ of leiomyoma of the urinary bladder in human is equivalent or slightly higher than muscle, and approximately $>40$ after contrast enhanced [12]. The most commonly seen clinical signs of urinary bladder leiomyoma are urinary incontinence and a palpable caudal abdominal mass [5,8].

Virtual autopsy refers to the postmortem use of radiology, ultrasound and cross-sectional imaging prior to conventional necropsy [11]. In this case report we demonstrate the detection of a rare extra-luminal urinary bladder mass diagnosed as leiomyoma with a postmortem ultrasound (PMUS) and postmortem computed tomography (PMCT) techniques.

\section{CASE}

The body of a 16-year-old male intact Schnauzer was presented for necropsy. The dog had previous history of seizure and no complains related to the urinary system. The animal was treated symptomatically to the neurological signs and responded to medical treatment. Nine weeks later from the first visit to the hospital the dog was found dead at home. The owner consented to donate the cadaver to participate in a virtual necropsy study in which both postmortem ultrasound (PMUS) and postmortem computed tomography (PMCT) were performed. A partial necropsy just to further assess the PMUS and PMCT findings was performed and a sample of the mass found in the urinary bladder was sent to histological examination.

Postmortem ultrasound of the abdomen was performed using a ultrasound system (MyLab ${ }^{\mathrm{TM}} 40$ VET $)^{1}$ with a linear transducer $(3.5-11 \mathrm{MHz})$. Whole body PMCT was performed with a CT helical multislice scanner (MX8000 IDT 16-row) ${ }^{2}$. The technical settings were $120 \mathrm{kVp}, 240 \mathrm{mAs}, 200 \mathrm{~mm}$ field of view, $512 \times 512$, and the study were acquired with a slice thickness of $3 \mathrm{~mm}$ and $1.5 \mathrm{~mm}$ of reconstruction interval. The study was acquired with the soft tissue algorithm and in helical mode.

Postmortem ultrasound revealed a homogenously hypoechoic (compared to the echogenicity of the urinary bladder wall), rounded and slightly irregularly marginated mass located externally but adjacent to the left cranial wall of the urinary bladder and appears to extend from its serosal margins (Figure 1). After administration of $10 \mathrm{~mL}$ of tap water via urinary bladder catheter, the mass was confirmed to originate from the muscularis layer of the urinary bladder wall. The mass measured approximately 2.9 $\mathrm{cm}$ in length by $1.4 \mathrm{~cm}$ in height by $2.3 \mathrm{~cm}$ in width.

Postmortem computed tomography was performed after PMUS. A pedunculated homogenous soft tissue attenuating mass $(\mathrm{HU}=72)$ was located at the left lateral aspect of the urinary bladder and extended cranially. It had a stalk that connected to the left lateral wall of the urinary bladder. The stalk was approximately $6 \mathrm{~mm}$ in diameter (Figure 2 ). The

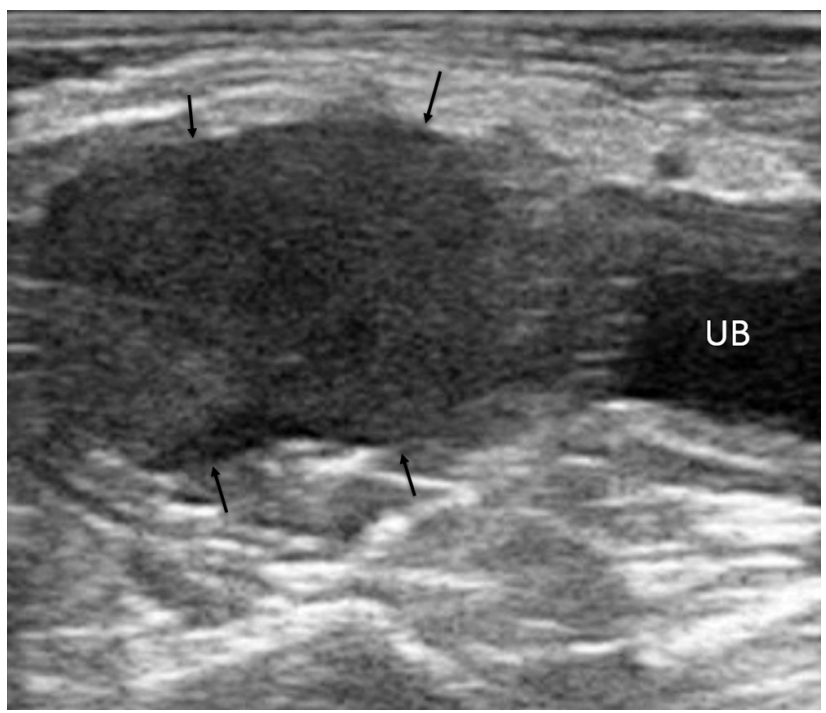

Figure 1. Ultrasound image of the mass: Transverse image of the homogenously hypoechoic extraluminal mass (between the black arrows) of the urinary bladder (UB). The urinary bladder is not fully distended; thus the wall appears thick. The stalk between the mass and the urinary bladder is not visualized, probable due to close proximity of both. 


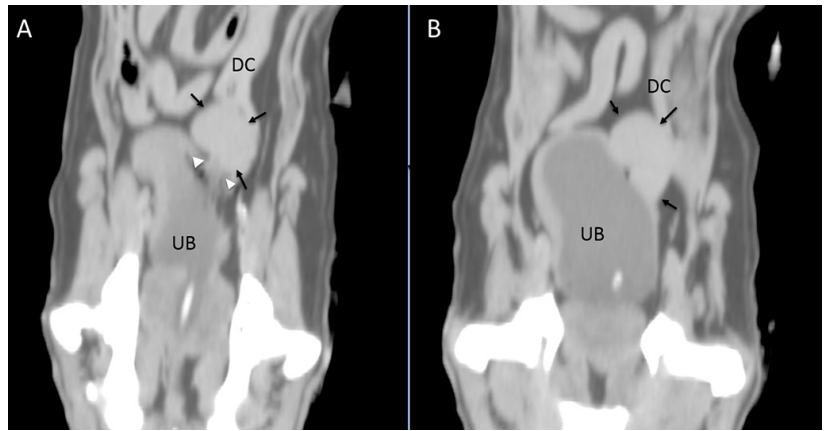

Figure 2. Computed tomography image of the mass: Dorsal reconstruction of PMCT study at two different level. Black arrows indicate the margins of the extraluminal, pedunculated soft tissue attenuating mass. White arrow heads demarcate the stalk of the mass. A- is located at a more dorsal level and B- is located at a more ventral level. The descending colon (DC) is located adjacent to the cranial and lateral margins of the mass. $\mathrm{UB}=$ urinary bladder.

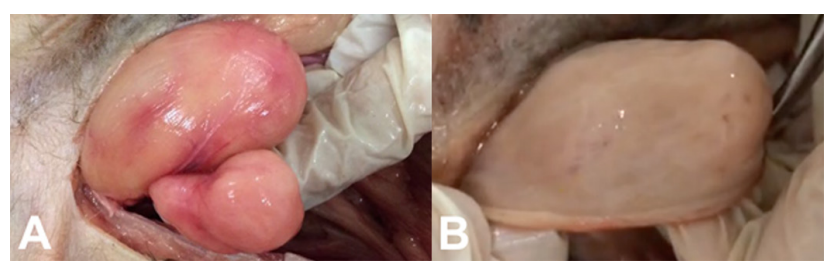

Figure 3. A- Gross necropsy shows the pedunculated mass originates from the left lateral wall of the urinary bladder. B- The mucosa of the urinary bladder is normal.

mass measured approximately $2.9 \mathrm{~cm}$ in length by $2.0 \mathrm{~cm}$ in height by $2.2 \mathrm{~cm}$ in width. The remaining findings of the PMCT were unremarkable.

A partial necropsy of the abdomen was done just to examine the mass. A round extraluminal, pedunculated mass was observed at the left lateral aspect of the urinary bladder wall. It was pale pink on the outside and white inside, with a soft to firm consistency (Figure 3a). The lumen and mucosal surface of the urinary bladder was smooth and regular (Figure 3b). The histology of the mass revealed a densely cellular neoplastic proliferation, expansive, composed of spindle-shaped cells with moderate to large eosinophilic cytoplasm, sometimes wavy and with indistinct edges. The nuclei were large, oval to flattened, with dense chromatin and inconspicuous nucleoli. Anisocytosis and anisokariosis were discrete and no mitotic figures were observed. The arrangement consisted of dense, irregular and multidirectional bundles and the stroma was scarce. The mass was histologically confirmed as leiomyoma. The stalk of the mass was not well represented in the histological block.

\section{DISCUSSION}

In this case, we performed PMUS and PMCT as part of a virtual necropsy study and in both modali- ties the urinary bladder mass was able be identified, followed by a partial necropsy to further investigate the nature of the mass and to collect a sample to obtain the histological diagnosis. A few of the disadvantages of the PMUS and PMCT specially in this case were the lack of color Doppler investigation on US and the lack of evaluation of the patter of contrast enhancement on CT. These techniques could have added important information related to the vascularity characteristics of the mass in a living patient.

This report describes an unusual extraluminal, pedunculated canine urinary bladder leiomyoma. The smooth and well-defined aspect of the mass margins was similar to previous report [7]. However, the mass of this report was homogenenously hypoechoic rather than heteroechoic [7]. The fluid administration to distend the urinary bladder helped to reduce the surface area of contact between the mass and the urinary bladder, making the identification of the origin of the mass possible. The PMCT helped to identify the stalk connecting the mass to the urinary bladder wall. The Hounsfield unit and homogenous attenuating nature of this mass is similar to reported cases in human. [5,8].

No history of incontinence was reported in this case, perhaps due to extraluminal and pedunculated nature of the mass. We failed to palpate this mass maybe due to its small size and also its close proximity to the colon.

Due to lack of complete history, the probable cause of death in this animal was not determined. As there were no abnormalities in the thorax or abdomen to explain the cause of death in this dog, recurrent neurological sign (seizures) that lead to death could not be ruled out. In our case, the unusual location of the urinary bladder tumor was the motivation for this report.

\section{MANUFACTURERS}

${ }^{1}$ Esaote Benelux B.V. Maastricht, The Netherlands. ${ }^{2}$ Philips Medical Systems. Highland Heights, OH, USA.

Acknowledgements. This work was financial supported by Capes Foundation - Coordenação de Aperfeiçoamento de Pessoal de Nível Superior - Process n. 99999.000199/2016-08. Ethical approval. This study was approved by ethics committee of the institution in the protocol 4720160216.

Declaration of interest. The authors declare no conflicts of interest. 
B. Ferrante, C.O. Baroni, C. Muramoto, et al. 2017. Post Mortem Ultrasound and Computed Tomography Findings of an Extraluminal Urinary Bladder Leiomyoma in a Dog.

Acta Scientiae Veterinariae. 45(Suppl 1): 237.

\section{REFERENCES}

1 Dere Y., Çelik S.Y., Çelik Ö.I., Deliktas H. \& Sahin H. 2015. Leiomyoma in a very rare location: bladder. Archivos Españoles de Urología. 68: 585-586.

2 Heng H.G., Lowry J.E., Boston S., Gabel C., Ehrhart N. \& Gulden S.M.S. 2006. Smooth muscle neoplasia of the urinary bladder wall in three dogs. Veterinary Radiology and Ultrasound. 47: 83-86.

3 Kabalin J.N., Freiha F.S. \& Niebel J.D. 1990. Leiomyoma of bladder report of 2 cases and demonstration of ultrasonic appearance. Urology. 35: 210-212.

4 Meuten D.J. 2002. Tumors of urinary system. In: Meuten D.J. (Ed). Tumors in Domestic Animals. 4th edn. Ames: Wiley Blackwell, pp.509-546.

5 Mouli S., Casalino D.D. \& Nikolaidis P. 2012. Imaging features of common and uncommon bladder neoplasms. $R a$ diologic Clinics of North America. 50: 301-316.

6 Nazir S.S., Maqbool A. \& Khan M. 2009. Leiomyoma of the urinary bladder - a case report and brief review of literature. International Journal of Nephrology \& Urology. 1: 72-74.

7 Norris A.M., Laing E.J., Valli V.E.O., Withrow S.J., Macy D.W., Ogilvie G.K., Tomlinson J., McCaw D., Pidgeon G. \& Jacobs R.M. 2008. Canine bladder and urethral tumors: a retrospective study of 115 cases (1980-1985). Journal of Veterinary Internal Medicine. 6: 145-153.

8 Seely J.C., Cosenza S.F. \& Montgomery C.A. 1978. Leiomyosarcoma of the canine urinary bladder, with metastases. Journal of the American Veterinary Medical Association. 172: 1427-1429.

9 Strafuss A.C. \& Dean M.J. 1975. Neoplasms of the canine urinary bladder. Journal of the American Veterinary Medical Association. 166: 1161-1163.

10 Tan K.Y., Yip S.K.H. \& Tan P.H. 2000. Clinics in diagnositc Imaging (50). Singapore Medical Journal. 4: 301-304.

11 Thali M.J., Yen K., Schweitzer W., Vock P., Boesch C., Ozdoba C., Schroth G., Ith M., Sonnenschein M., Doernhoefer T., Scheurer E., Plattner T. \& Dirnhofer R. 2003. Virtopsy, a new imaging horizon in forensic pathology: virtual autopsy by postmortem multislice computed tomography (MSCT) and magnetic resonance imaging (MRI)-a feasibility study. Journal of Forensic Sciences. 48: 386-403.

12Zhang L.Y. \& Dai J.R. 2009. Imaging features of nonepithelial tumors of the bladder. Chinese Journal of Oncology. 31: 384-387. 